\title{
Desenvolvimento de Nanocompósitos Polipropileno/Argila Bentonita Brasileira: I Tratamento da Argila e Influência de Compatibilizantes Polares nas Propriedades Mecânicas
}

\author{
André W. Rodrigues \\ Programa de Pós-Graduação em Ciência e Engenharia de Materiais, UFCG \\ Maria I. Brasileiro \\ Programa de Pós-Graduação em Engenharia Química, UFCG \\ Williane D. Araújo, Edcleide M. Araújo, Gelmires A. Neves, Tomás J. A. de Melo \\ Engenharia de Materiais, UFCG
}

\begin{abstract}
Resumo: Os nanocompósitos de polipropileno/ bentonita e polipropileno/compatibilizante/bentonita foram preparados empregando uma extrusora dupla rosca acoplada a um Reômetro de Torque Haake. A argila bentonita natural proveniente do Município de Boa Vista - PB passou por um processo de purificação para em seguida ser aditivada com carbonato de sódio e posteriormente tratadas com o sal quaternário de amônia, visando a obter argila organofílica. As argilas foram caracterizadas por granulometria por difração de laser, análise química, espectroscopia de infravermelho e DR-X. Os resultados de granulometria e análise química mostraram que o processo de purificação foi eficiente em retirar as frações mais grosseiras mantendo as frações finas bem como na eliminação de minerais acessórios. Os resultados de FTIR e DR-X mostraram que o sal quaternário de amônio foi incorporado à argila confirmando a organofilização. Posteriormente a argila bentonita organofílica foi incorporada ao polipropileno (PP) e PP/compatibilizante (PP-g-AA e PP-g-MA são polipropilenos enxertados com ácido acrílico e anidrido maleico, respectivamente). A formação dos nanocompósitos bem como as propriedades das misturas PP/ORG (ORG - argila organofílica) e PP/compatibilizante/ORG foram verificadas por DR-X, MET e propriedades mecânicas. As análises de DR-X, MET mostraram que pode ter ocorrido a formação de nanocompósitos intercalado devido aos deslocamentos dos picos para ângulos de $2 \theta$ inferiores. Os resultados das propriedades mecânicas mostraram que houve uma melhoria em algumas propriedades mecânicas.
\end{abstract}

Palavras-chave: Argila bentonita, nanocompósito, compatibilizante, polipropileno.

\section{Development of Polypropylene/Brazilian Bentonite Clay Nanocomposites: I Treatment of Clay and the Influence of the Compatibilizers on the Mechanical Properties}

Abstract: In this work, polypropylene/bentonite and polypropylene/compatibilizer/bentonite nanocomposites were prepared by the melt intercalation method, using a counter-rotating intermeshing twin-screw extruder attached to a Haake System 90 Rheometer. Initially the natural bentonite clay, from Boa Vista - PB, was subjected to a purification process and then treated with sodium carbonate. Further, it was treated with ammonium quaternary salt to obtain an organoclay. The clays were characterized, according to granulometric, by laser diffraction, chemical analysis, infrared spectroscopy - FTIR, and $\mathrm{X}$ ray diffraction $-\mathrm{XRD}$. The granulometric and chemical analysis results showed that the purification process was effective for removing the coarse fractions and eliminating some accessory minerals. FTIR and XRD results showed that the ammonium quaternary salt was incorporated to the clay, confirming the organophilization. In the subsequent stage of this work, the bentonite organoclay was incorporated to both polypropylene (PP) and PP/compatibilizer (PPgAA and PPgMA are polypropylenes grafting acrylic acid and maleic anhydride, respectively). The formation of nanocomposites as well as the properties of PP/ORG (ORG - organoclay) and PP/compatibilizer/ORG mixtures were analyzed by XRD, MET and mechanical properties. XRD and MET results showed that the formation of interlayer/flocculated nanocomposites has occurred for PP/compatibilizer/ORG systems. The mechanical properties results showed that some mechanical properties (final elongation and toughness) have been considerably improved.

Keywords: Nanocomposite, organofilization, compatibilizer, polypropylene.

Autor para correspondência: Tomás J. A. de Melo, Departamento de Engenharia de Materiais, CCT, UFCG, Rua Aprígio Veloso 882 - Bodocongó, CEP: 58109-970, Campina Grande, PB, Brasil. E-mail: tomas@dema.ufcg.edu.br 


\section{Introdução}

O campo de aplicação dos polímeros tem sido largamente ampliado nos últimos anos, ocupando espaços antes pertencentes aos outros materiais como às cerâmicas e os metais. Estas novas aplicações requerem, necessariamente, novas propriedades que muitas vezes o polímero puro não possui. Umas das formas de modificar as propriedades dos polímeros é por meio da incorporação de cargas (talco, carbonato de cálcio, caulim, fibras de vidro, etc) neste caso, o material é denominado de compósito polimérico. Recentemente uma nova classe de compósitos tem sido desenvolvido os nanocompósitos poliméricos ${ }^{[1]}$. Os nanocompósitos são materiais nos qual a fase dispersa apresenta pelo menos uma dimensão de ordem nanométrica ${ }^{[2]}$. A utilização de nanocargas permite o melhoramento das propriedades de barreira, estabilidades térmica e dimensional, retardância de chama e propriedades mecânicas com emprego de baixos teores de carga (até 5\% em massa) o que os diferencia dos compósitos tradicionais que utilizam até $40 \%$ em peso $^{[3-6]}$. Os nanocompósitos polímero/argila tiveram maior importância de aplicação industrial a partir do desenvolvimento por um grupo de pesquisadores da Toyota $^{[3]}$. Eles obtiveram nanocompósitos de nylon-6/argila organofílica por meio do processo de polimerização in situ da $\varepsilon$-caprolactona. $O$ resultado foi uma significativa melhoria nas propriedades quando comparados às do polímero puro ${ }^{[3]}$.

Vários métodos de obtenção de nanocompósitos poliméricos foram desenvolvidos, polimerização in situ, em solução e intercalação por fusão. Dentre eles, o método de intercalação por fusão tem sido mais empregado, pois é possível obter nanocompósitos poliméricos sem a utilização de solventes, reduzindo o numero de etapas, riscos ambientais e custo $^{[6-8]}$. Além disso, é possível utilizar equipamentos já disponíveis para mistura e processamento de polímeros, como extrusoras e injetoras ${ }^{[3]}$.

A bentonita, que tem como argilomineral predominante a montmorilonita, é a argila mais comumente usada na obtenção de nanocompósitos polímero/argila, sendo que deve ser modificada organicamente com sais quaternários de amônio para melhorar a sua interação com a matriz polimérica ${ }^{[9-11]}$. Grande parte das reservas nacionais de bentonita, cerca de $62 \%$, estão localizadas no estado da Paraíba, nos municípios de Boa Vista e Cubati. Enquanto que o estado de São Paulo possui cerca de $28 \%$ e os estados da Bahia, Minas Gerais e Paraná ficam com os $10 \%$ restantes $^{[12]}$.

Diversos polímeros estão sendo empregados como matriz na preparação de nanocompósitos polímero/argila. O polipropileno (PP) é um dos polímeros mais usados pois exibe uma atrativa combinação de baixo custo e grande versatilidade em termos de propriedades, aplicações e reciclagem ${ }^{[13]}$. Contudo, este polímero não possui nenhum grupo polar em sua cadeia que possa interagir com grupos polares presentes nas superfícies das argilas usadas como cargas. A ausência de polaridade no PP dificulta a interação e intercalação das cadeias poliméricas entre as lamelas das partículas de argila. Visando sintetizar nanocompósitos PP/argila, alguns estudos foram realizados com matriz de PP misturado com o PP enxertado com grupos polares e com argila organofílica. A finalidade de utilizar o PP enxertado com grupos polares, por exemplo: do tipo anidrido maleico e ácido acrílico, é para promover interações químicas destes grupos, por meio de pontes de hidrogênio, com as hidroxilas presentes na superfície da argila e facilitar o processo de intercalação ${ }^{[13-15]}$.

Neste trabalho o objetivo principal foi o desenvolvimento de nanocompósitos com matriz polimérica de polipropileno misturada com argila bentonita brasileira. Para a obtenção dos nanocompósitos foram realizados dois tratamentos na argila (purificação e organofilização) e avaliada a influência de compatibilizantes polares nas propriedades mecânicas.

\section{Experimental}

\section{Materiais}

- Polipropileno (PP), código RP 347, em grânulos. Com $\mathrm{IF}=10 \mathrm{~g} / 10 \mathrm{~min}\left(2,16 \mathrm{~kg} / 230^{\circ} \mathrm{C}-\right.$ ASTMD-1238-L $)$ fabricado e doado pela Braskem S.A;

- Argila bentonítica natural $(\mathrm{N})$, variedade identificada industrialmente de "chocolate", proveniente da Mina Bravo, localizada no município de Boa Vista - PB;

- Sal quaternário de amônio, tipo Dodigen ${ }^{\circledR} 1611$, fornecido pela Clariant Brasil. Sua estrutura molecular está apresentada a seguir:

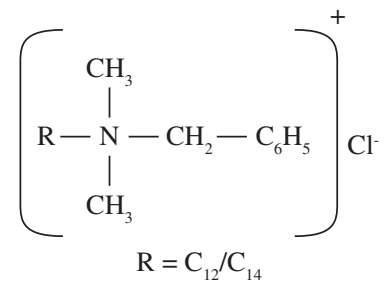

- Polipropileno enxertado com ácido acrílico (PP-g-AA) polybond 1001, IF = $40 \mathrm{~g} / 10 \mathrm{~min}$, concentração de ácido: $6 \%$ em peso, fabricado pela Uniroyal Chemical e fornecido pela Crompton; e

- Polipropileno enxertado com Anidrido Maleico (PP-gMA) polybond $3200, \mathrm{IF}=110 \mathrm{~g} / 10 \mathrm{~min}$, concentração de anidrido: $2,7 \%$ em peso, fabricado pela Uniroyal Chemical e fornecido pela Crompton.

\section{Tratamento da argila: purificação e organofilização}

\section{Purificação}

Utilizou-se a argila bentonita natural $(\mathrm{N})$, variedade identificada como chocolate, proveniente do município de Boa Vista - PB. Na primeira etapa, a purificação, a argila natural, passou por um processo de secagem e desagregação. Em seguida, foi dispersa em água deionizada tratada com $20 \mathrm{ml}$ hidróxido de amônio $\left(\mathrm{NH}_{3} \mathrm{OH}\right)$ e deixada em repouso por 7 dias. Após os 7 dias a amostra foi agitada em agitadores a velocidade de $17.000 \mathrm{rpm}$ por 30 min e colocada em um recipiente para sedimentação seletiva, permanecendo em repouso por 24 horas. 
Após este período, foi coletado do tanque um volume correspondente aos 2/3 superiores da dispersão. Esta sistemática consiste no processo de purificação, que objetiva a eliminação de acessórios na forma de matéria orgânica e partículas de quartzo (areias e siltes), mica e outros argilominerais tais como caulinita e ilita bem como separação granulométrica visando obter partículas de argilas com dimensões nanométricas ${ }^{[16,17]}$.

A dispersão então foi mantida em temperatura de $60{ }^{\circ} \mathrm{C}$ para que a concentração de sólidos atinja o valor de $4,86 \%$, sendo em seguida aditivada com uma solução de carbonato de sódio $\left(\mathrm{Na}_{2} \mathrm{CO}_{3}\right)$ com concentração de $0,2 \mathrm{~g} / \mathrm{ml}$ na proporção de 100 meq de $\mathrm{Na}_{2} \mathrm{CO}_{3} / 100 \mathrm{~g}$ de argila seca, permanecendo em cura por 7 dias. Nesta etapa a argila purificada é tornada predominantemente sódica (SOD).

\section{Organofilização}

$\mathrm{Na}$ segunda etapa a organofilização, a argila bentonita sódica (SOD) foi tratada com o sal Dodigen 1611, com o seguinte procedimento: as dispersões com concentração de $4,86 \%$ foram separadas em volumes de $400 \mathrm{~mL}$ e tratadas com $12,31 \mathrm{~g}$ do sal quaternário de amônio. A agitação foi mantida por 20 minutos. Feito isso, os recipientes foram fechados e mantidos à temperatura ambiente por 24 horas. Após esse tempo, o material obtido foi filtrado a vácuo para remoção do excesso de sal. A lavagem foi feita com $2000 \mathrm{~mL}$ de água deionizada, empregando-se funil de Buchner com kitassato, acoplado a uma bomba de vácuo, onde foi utilizado papel de filtro qualitativo e vácuo com $700 \mathrm{mmHg}$. Os aglomerados obtidos foram secados em estufa a $60 \pm 5{ }^{\circ} \mathrm{C}$. Por fim, os aglomerados secados foram desagregados com o auxílio de almofariz até obter materiais pulverulentos os quais foram passados em peneira ABNT $n^{\circ} 200(\mathrm{D}=0,074 \mathrm{~mm})$ para em seguida serem caracterizados ${ }^{[18]}$.

\section{Preparação dos nanocompósitos com dois tipos de compatibilizantes}

Inicialmente a argila bentonita sódica (SOD) e a argila bentonita organofílica (ORG), foram secadas em estufa na temperatura a $60{ }^{\circ} \mathrm{C}$ durante 24 horas. Em seguida, misturadas em concentração de $50 \%$ em peso com o polipropileno no misturador fechado do tipo Banbury na temperatura de $210{ }^{\circ} \mathrm{C}$ e velocidade dos rotores de $60 \mathrm{rpm}$, durante $10 \mathrm{mi}-$ nutos. O concentrado resultante "masterbatch" foi posteriormente, triturado e misturado com o PP, com o PP/PP-g-AA (90/10) e com o PP/PP-g-MA (90/10) em concentrações de 1 e $3 \%$ em peso de argila numa extrusora com rosca dupla contra-rotativa, acoplada a um reômetro de torque System 90 da Haake-Blucher, nas seguintes condições: temperatura do cilindro de $180 / 210 / 210 / 210 / 210{ }^{\circ} \mathrm{C}$ (da alimentação à matriz), velocidades de rotação da rosca de $60 \mathrm{rpm}$ e alimentação constante. Os extrudados após resfriamento foram granulados e moldados por injeção, na mesma temperatura de extrusão $\left(210^{\circ} \mathrm{C}\right.$ em todas as zonas da injetora), na forma de corpos de prova de tração e impacto, segundo normas ASTM D638 e D256, respectivamente.

\section{Caracterização das argilas}

\section{Análise química (AQ)}

A análise química das argilas foi realizada segundo os métodos clássicos (determinações gravimétricas, volumétricas e colorimétricas) e métodos instrumental e complexométrico (determinações espectrofotométricas e por fotometria de chama) de acordo com a descrição encontrada em Sousa Santos ${ }^{[24]}$.

\section{Difração de raios X (DR-X)}

Para analisar e comparar o grau de intercalação do sal quaternário de amônio, tipo Dodigen 1611, foi utilizado o difratômetro de raios $\mathrm{X}$ da marca Shimadzu (radiação $\mathrm{Cu} \mathrm{K} \alpha$ ) operando na faixa de $2 \theta$ de 0 a 30 graus.

\section{Espectroscopia de infravermelho (FTIR)}

As argilas foram analisadas através de espectroscopia de infravermelho em equipamento modelo AVATAR TM 360 NICOLET. As condições de operação foram: região $400-4000 \mathrm{~cm}^{-1}$, com 10 varreduras e resolução de $4 \mathrm{~cm}^{-1}$. As amostras de bentonita foram caracterizadas na forma de pastilha feitas a partir de 0,007 $\mathrm{g}$ de argila e $0,1 \mathrm{~g}$ de $\mathrm{KBr}$ prensadas a 5 toneladas por 30 segundos.

\section{Análise granulométrica por difração de laser (DL)}

A análise granulométrica por difração de laser utiliza o método de dispersão de partículas em fase líquida associado com um processo de medida óptica através de difração de laser. Neste método, é combinada a relação proporcional entre a difração do laser e a concentração e tamanho de partículas.

Para realização desta caracterização, a argila bentonítica foi peneirada através de peneira ABNT n ${ }^{\circ} 200(0,074 \mathrm{~mm})$, e dispersa em uma solução $250 \mathrm{~mL}$ de água destilada em agitador Hamilton Beach N5000 a velocidade de 17.000 rpm por 10 min. Em seguida, esta dispersão foi colocada em um equipamento CILAS modelo 1064, em via úmido, até atingir a concentração ideal que é de 150 unidades de difração/área de incidência.

\section{Caracterização dos nanocompósitos}

\section{Difração de raios X (DR-X)}

Para analisar e comparar o grau de intercalação e/ou de esfoliação (separação das camadas das partículas precursoras de argila nos nanocompósitos desenvolvidos por extrusão), foi utilizado o difratômetro de raios X da marca Shimadzu (radiação $\mathrm{Cu} \mathrm{k} \alpha$ ) operando na faixa de $2 \theta$ de 0 a 30 graus.

\section{Microscopia eletrônica de transmissão (MET)}

O microscópio eletrônico de transmissão utilizado foi da marca PHILIPS CM120 pertencente à UFSCar operando a uma voltagem de aceleração de $120 \mathrm{KV}$. As amostras foram retiradas do centro do corpo de prova de impacto. Foram preparadas através da redução de área pelo procedimento do 
“trimming”, em forma trapezoidal com área de aproximadamente $0,5 \mathrm{~mm}^{2}$. Os cortes das amostras foram realizados em um ultramicrótomo da marca RMC modelo MT-7000 usando uma faca de diamante da marca Diatome tipo Cryohisto $45^{\circ}$. As condições de corte foram: temperatura de $-80{ }^{\circ} \mathrm{C}$, na amostra e na faca de diamante, resfriamento com nitrogênio liquido, e velocidade de corte de $0,1 \mathrm{~mm} / \mathrm{s}$. A espessura das amostras ficou em torno de 25 e $50 \mathrm{~nm}$.

\section{Propriedades mecânicas}

Os ensaios foram feitos em uma máquina universal de ensaios LLOYD INSTRUMENTS LR 10KN, operando a uma velocidade de deformação de $50 \mathrm{~mm} / \mathrm{min}$, em corpos de prova do tipo I, segundo norma da ASTM D 638. Este ensaio foi com uma media de $10 \mathrm{CP}$.

\section{Resultados e Discussão}

\section{Caracterização das argilas purificada e organofilica}

\section{Análise química}

Na Tabela 1 estão apresentados os resultados da composição química das argilas chocolate (natural) e purificada, o desvio-padrão obtido da análise de três amostras foi inferior a 5\%. Analisando os dados da Tabela observam-se variações em: PR e teores de $\mathrm{Fe}_{2} \mathrm{O}_{3}, \mathrm{MgO}, \mathrm{K}_{2} \mathrm{O}$ e RI após o tratamento. $\mathrm{O}$ aumento de PR deve-se provavelmente a diminuição de resíduo insolúvel o que aumenta a quantidade de fração argila e consequientemente a perda ao rubro. A redução do teor de sílica deve-se provavelmente a eliminação de sílica livre na forma de grão de quartzo após o processo de purificação. $\mathrm{O}$ aumento de $\mathrm{Al}_{2} \mathrm{O}_{3}$ de 21,35 para 25,33 deve-se provavelmente a uma maior concentração da fração argila. A redução do teor de $\mathrm{MgO}$ e RI, deve-se provavelmente, a sedimentação das partículas após o período de repouso de 24 horas. Para o $\mathrm{Fe}_{2} \mathrm{O}_{3}$, a amostra purificada apresentou teor de 5,59\% valor inferior ao apresentado pela amostra natural. Este decréscimo deve-se provavelmente a eliminação, através da purificação, de argilominerais não motmoriloníticos, uma vez que as argilas de Boa Vista têm teores de $\mathrm{Fe}_{2} \mathrm{O}_{3}$ superiores a 7\%. Os demais teores contidos na Tabela 1 mostram uma composição química típica das argilas bentoniticas de Boa Vista - PB.

\section{Espectroscopia de absorção no infravermelho (FTIR)}

Na Figura 1 estão apresentados os espectros na região do infravermelho das argilas: purificada, sódica (SOD) e organofílica (ORG). Nesta figura os espectros foram deslocados na vertical para melhorar a comparação entre eles.

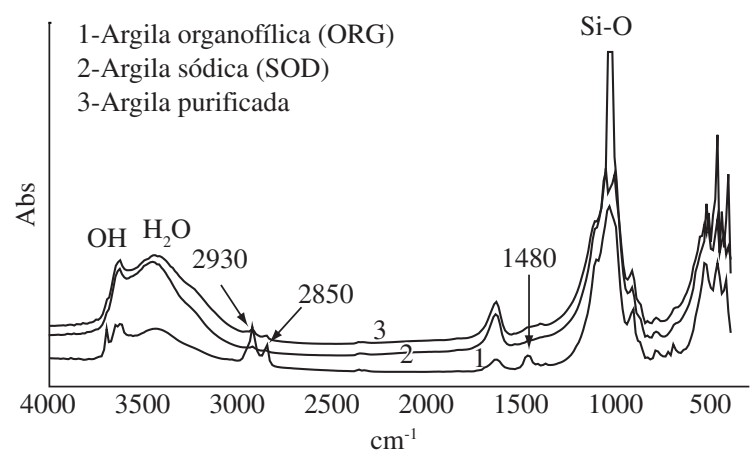

Figura 1. Espectros no Infravermelho das argilas: purificada; sódica (SOD); e organofílica (ORG). (Abs- absorbância).

O espectro de absorção na região do infravermelho da argila após o tratamento com o sal quaternário de amônio, revela a presença de novas bandas: a $2930 \mathrm{~cm}^{-1}$, referente às vibrações de deformação axial assimétrica dos grupos $\mathrm{CH}_{3} \mathrm{e}$ $\mathrm{CH}_{2}$; a $2850 \mathrm{~cm}^{-1}$, referente às vibrações de deformação axial simétrica dos grupos $\mathrm{CH}_{3}$ e $\mathrm{CH}_{2}$ e a $1480 \mathrm{~cm}^{-1}$, referente às vibrações de deformação angular assimétrica e simétrica dos grupos $\mathrm{CH}_{3}$ e $\mathrm{CH}_{2}$, respectivamente. Estes grupos fazem parte da estrutura química do sal o que demonstra a presença do sal na argila. Estes resultados estão de acordo com os resultados obtidos por outros autores que utilizaram à mesma argila (natural) e o mesmo método de organofilização ${ }^{[12]}$.

\section{Difração de raios X - DRX}

Na Figura 2 estão apresentados os espectros de difração de raios X das argilas: natural, purificada, sódica (SOD) e organofílica (ORG). Observam-se que nas argilas natural, purificada e sódica a montmorilonita $(\mathrm{M})$ está presente devido o surgimento de reflexões do plano $\left(\mathrm{d}_{001}\right)$ em $2 \theta \sim 6$ e $7^{\circ}$. As demais reflexões indicam a presença de quartzo(Q) com distâncias interplanares de 3,24, 3,35 e 4,24 Á e caulinita (C),

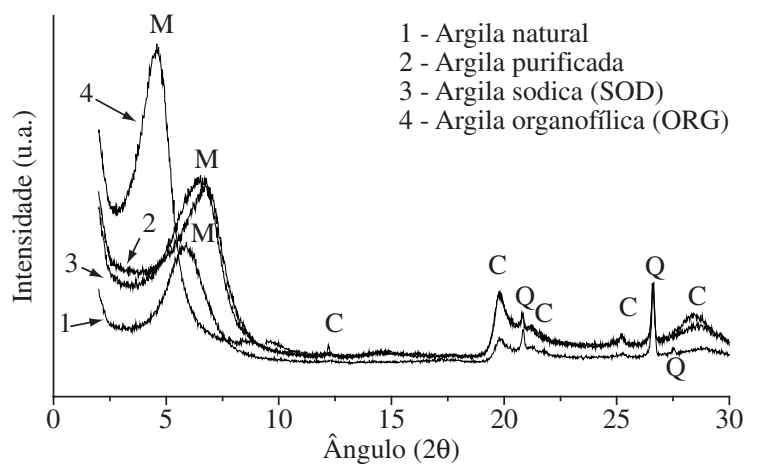

Figura 2. Difração de raios $X$ das argilas: 1- natural; 2- purificada; 3- sódica (SOD); e 4- organofílica (ORG).

Tabela 1. Análise química das argilas natural e purificada.

\begin{tabular}{ccccccccccc}
\hline Amostra da argila & $\mathrm{Tipo}$ & $\mathrm{PR}(\boldsymbol{\%})$ & $\mathbf{S i O}_{\mathbf{2}}(\boldsymbol{\%})$ & $\mathbf{A l}_{\mathbf{2}} \mathbf{O}_{\mathbf{3}}(\boldsymbol{\%})$ & $\mathbf{F e}_{\mathbf{2}} \mathbf{O}_{\mathbf{3}}(\boldsymbol{\%})$ & $\mathbf{C a O}(\boldsymbol{\%})$ & $\mathbf{M g O}(\boldsymbol{\%})$ & $\mathbf{N a}_{\mathbf{2}} \mathbf{O}(\boldsymbol{\%})$ & $\mathbf{K} \mathbf{O}(\%)$ & $\mathbf{R I}(\%)$ \\
\hline Chocolate & $\mathrm{N}$ & 11,56 & 54,74 & 21,35 & 8,00 & Traços & 2,02 & 0,50 & 0,12 & 1,06 \\
Chocolate & $\mathrm{P}$ & 13,08 & 53,66 & 25,33 & 5,59 & Traços & Traço & 0,55 & 0,05 & 0,70 \\
\hline
\end{tabular}

$\mathrm{N}$ - natural; P -purificada; PR - perda ao rubro; e RI - resíduo insolúvel. 
Tabela 2. Distância Interplanar basal $\mathrm{d}_{001}$ das amostras de argilas

\begin{tabular}{lcc}
\hline Amostras (argila chocolate) & $\mathbf{2 \theta}$ & $\mathbf{d}_{(\mathbf{0 0 1})}(\mathbf{A})$ \\
\hline Argila natural & 6,30 & 14,02 \\
Argila purificada & 6,36 & 13,68 \\
Argila sódica (SOD) & 6,60 & 13,36 \\
Argila organofílica (ORG) & 4,56 & 19,33 \\
\hline
\end{tabular}

em menor quantidade, com distâncias de 7,15, 4,48, 4,17, 3,52 e 3,08 Â. A eliminação de um pico de quartzo evidencia que a purificação eliminou apenas as frações mais grosseiras dos minerais acessórios, porém o quartzo na forma coloidal permanece, conforme evidenciado pelos picos de difração. Observou-se também que houve uma pequena redução no espaçamento interlamelar da montmorilonita (M) de 14,02 para 13,68 Á que tem como causa provável a presença de substâncias orgânicas (hidróxido de amônio) que foram eliminadas após a secagem da argila natural. Para a argila organofílica, houve um significativo deslocamento do pico característico da montmorilonita para um ângulo $2 \theta$ mais baixo indicando distâncias interlamelares do plano $\left(\mathrm{d}_{001}\right)$ superiores, em mais de $40 \%$ aos das bentonitas purificada e sódica, sugerindo que houve intercalação das moléculas do sal quaternário de amônio entre as lamelas da bentonita, conforme valores calculados e apresentados na Tabela 2.

\section{Análise granulométrica por difração de laser}

A Figura 3 apresenta o resultado da análise granulométrica da argila chocolate no estado natural (não purificada) tendo sido apenas peneirada em malha $200(0,074 \mathrm{~mm})$ e dispersa em água. Nestas condições o diâmetro médio das partículas é de 3,21 $\mu \mathrm{m}$. Observa-se que $44 \%$ da massa acumulada têm diâmetro médio equivalente abaixo de $2 \mu \mathrm{m}$, que corresponde a fração argila. E a maior concentração de partículas en-

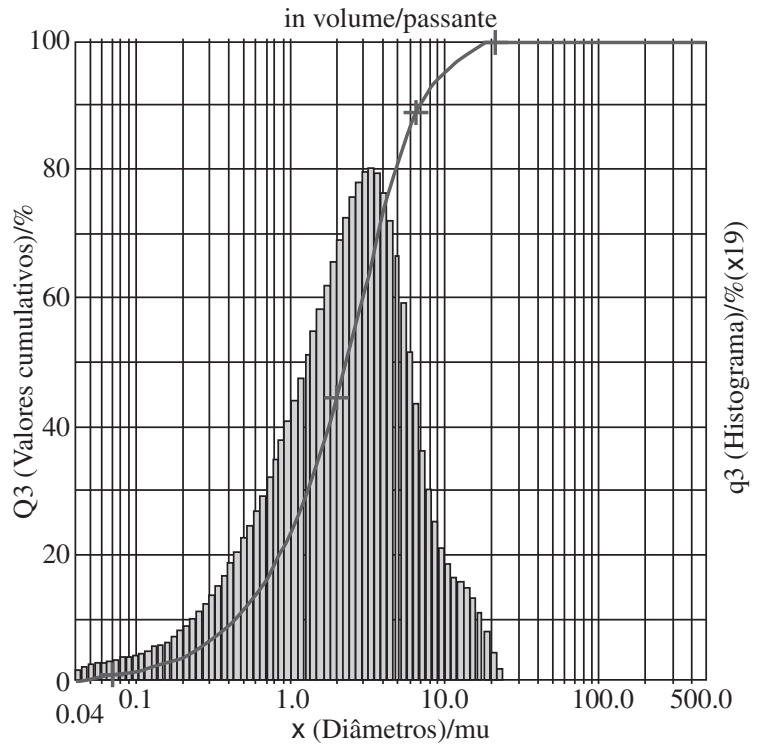

(a) tre 2 e $5 \mu \mathrm{m}$. A Figura $3 \mathrm{~b}$ mostra os resultados da análise granulométrica da argila chocolate purificada e tratada com hidróxido de amônia (agente dispersante). Depois do processo de purificação observa-se uma redução das frações finas abaixo de $0,2 \mu \mathrm{m}$ e maiores que $10 \mu \mathrm{m}$, estes cortes devem-se provavelmente ao processo de separação granulométrica por sedimentação na purificação da argila. As frações finas foram reduzidas por não ter sido possível recolher com a bomba de separação por sucção. E as frações grosseiras ficaram depositadas no fundo do tanque de decantação. Observa-se um diâmetro médio de $3,44 \mu \mathrm{m}$ e a maior concentração de partículas entre 3 e $7 \mu \mathrm{m}$. A ausência das frações maiores que $10 \mu \mathrm{m}$ não garante uma maior finura das partículas visto que no processo de purificação pode-se eliminar a fração coloidal mais fina que fica situada na camada superior do tanque de decantação, embora os resultados sejam muito próximos.

\section{Caracterização dos nanocompósitos}

\section{Difração de raios X (DR-X)}

A Figura 4 apresenta os difratogramas de DRX dos sistemas PP/ORG, PP/PP-g-AA/ORG e PP/PP-g-MA/ORG com teores de argila purificada de 1 e $3 \%$ em peso. Os resultados mostraram que as distâncias interplanares basais $\left(\mathrm{d}_{001}\right)$ dos sistemas acima, com 1 e $3 \%$ em peso, ficaram dentro de uma faixa de 16 a 17,5 $\AA$ de acordo com os dados apresentados na Tabela 3. Estes valores encontram-se entre a distância interplanar $\left(\mathrm{d}_{001}\right)$ da argila sódica $(13,36 \AA)$ e o da argila organofílica $(19,33 \AA)$ e em geral mostraram-se independentes dos teores de argila organofílica e da presença e do tipo de compatibilizante. Apesar de se obter valores de d001 intermediários pode ter ocorrido intercalação do polímero entre as lamelas da argila e esfoliação parcial das partículas, uma vez que os picos mostraram-se mais alargados em direção a

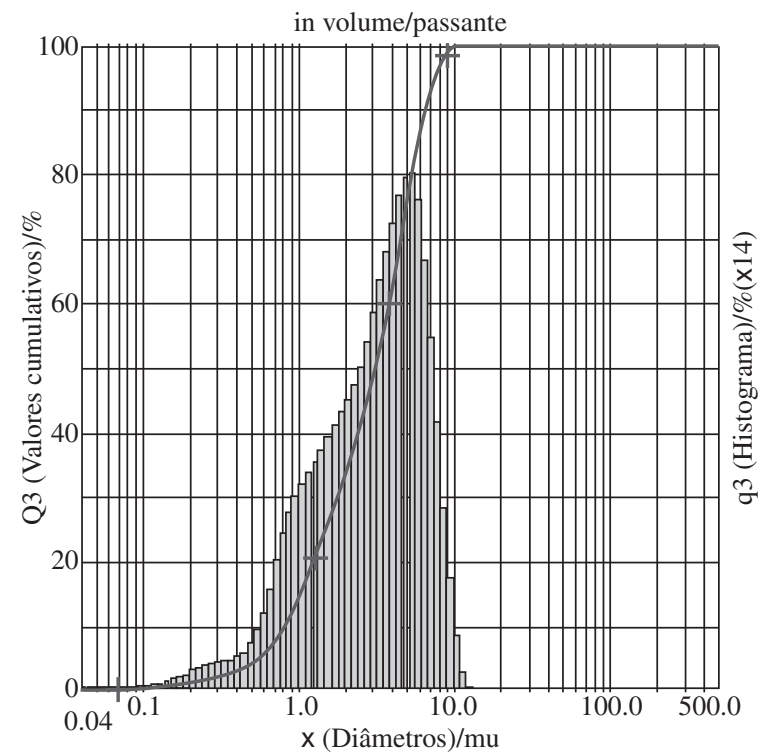

(b)

Figura 3. Resultado da análise granulométrica: a) argila natural; e b) argila purificada. 


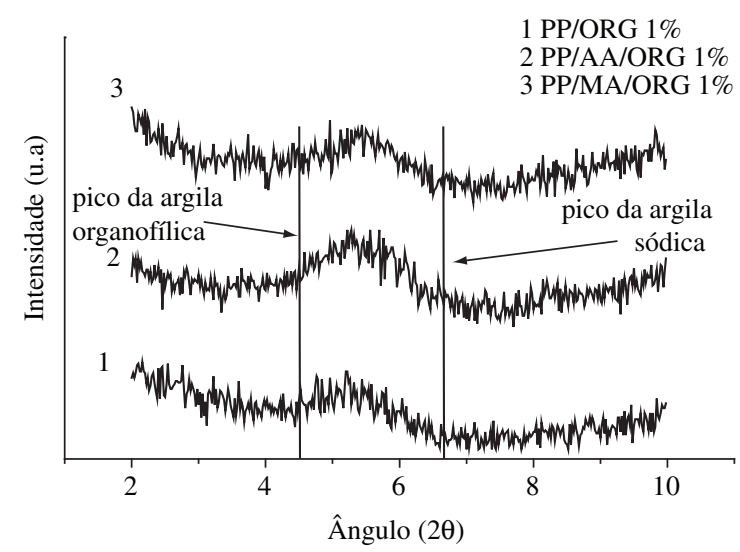

(a)

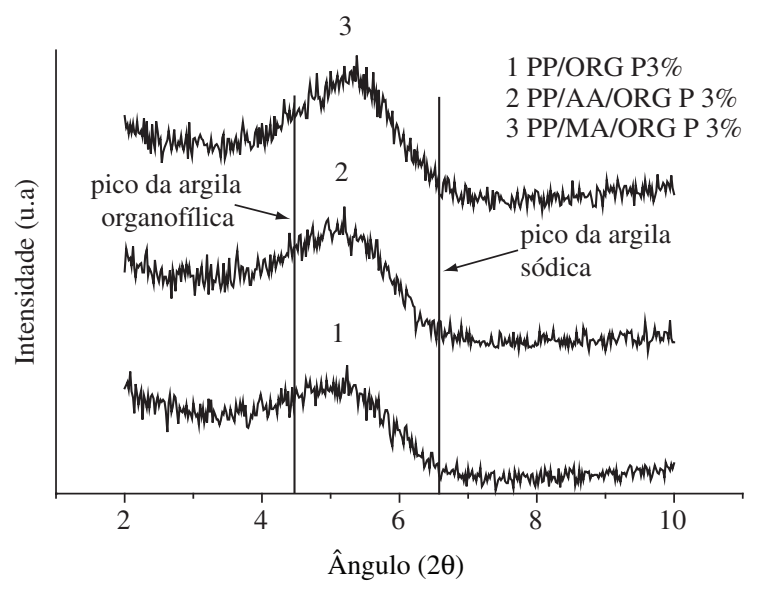

(b)

Figura 4. Difração de raios $X$ dos sistemas: a) PP/ORG 1\%, PP/PP-g-AA/ ORG $1 \%$ e PP/PP-g-MA/ORG $1 \%$; e b) PP/ORG 3\%, PP/PP-g-AA/ORG $3 \%$ e PP/PP-g-MA/ORG $3 \%$.

Tabela 3. Valores da distância interplanar basal $\left(\mathrm{d}_{001}\right)$.

\begin{tabular}{|c|c|c|c|c|}
\hline Amostras & \multicolumn{2}{|c|}{$2 \theta$} & \multicolumn{2}{|c|}{$d_{(001)} \AA$} \\
\hline Argila Sódica (SOD) & \multicolumn{2}{|c|}{6,60} & \multicolumn{2}{|c|}{13,36} \\
\hline Argila Organofílica (ORG) & \multicolumn{2}{|c|}{4,56} & \multicolumn{2}{|c|}{19,33} \\
\hline \multicolumn{5}{|c|}{ Sistemas com argila organofílica (ORG) } \\
\hline & \multicolumn{2}{|c|}{$1(\%)$} & \multicolumn{2}{|c|}{$3(\%)$} \\
\hline Amostras & $2 \theta$ & $d_{(001)} \AA$ & $2 \theta$ & 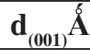 \\
\hline PP/ORG & 5,15 & 17,14 & 5,26 & 16,78 \\
\hline PP/PP-g-AA/ORG & 5,29 & 16,67 & 5,06 & 17,45 \\
\hline PP/PP-g-MA/ORG & 5,40 & 16,34 & 5,36 & 16,47 \\
\hline
\end{tabular}

$2 \theta$ menor, quando comparados com a argila organofílica. No item a seguir, estão apresentadas as micrografias dos sistemas com a argila purificada, obtidas por microscopia eletrônica de transmissão, e mostram evidências deste comportamento. Outros autores também observaram este fenômeno ${ }^{[19-21]}$.

\section{Microscopia eletrônica de transmissão (MET)}

A microscopia eletrônica de transmissão (MET) permite analisar em detalhes a morfologia dos nanocompósitos. A Figura 5 apresenta as fotomicrografias dos sistemas

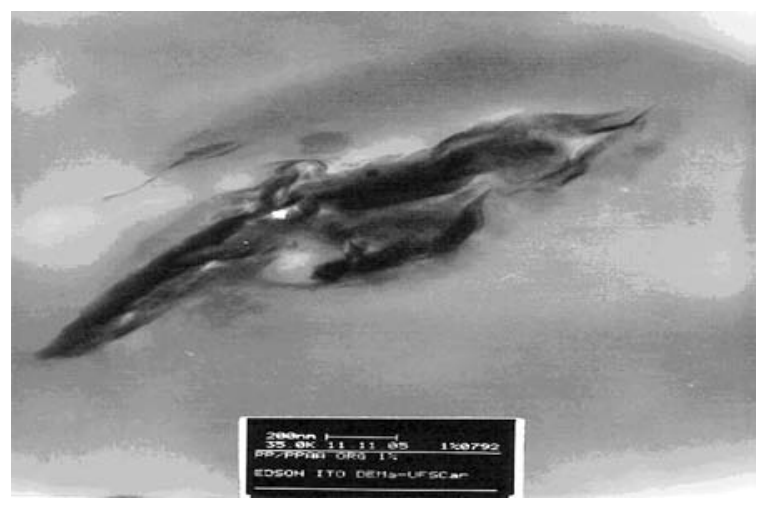

(a)

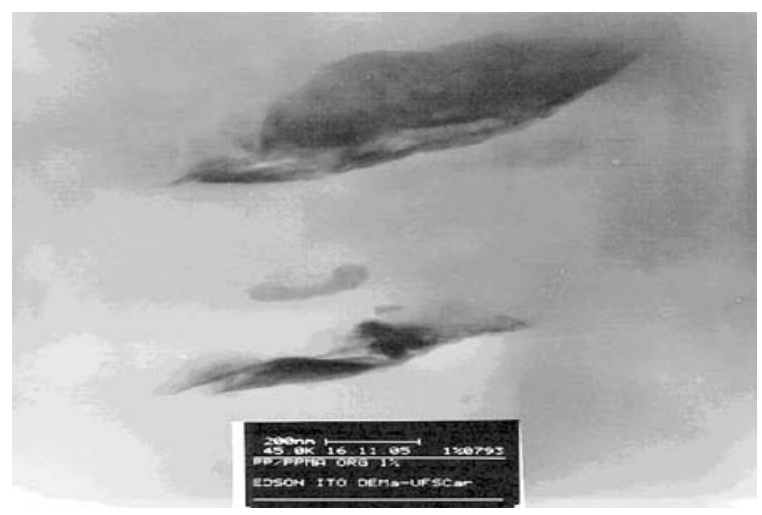

(b)

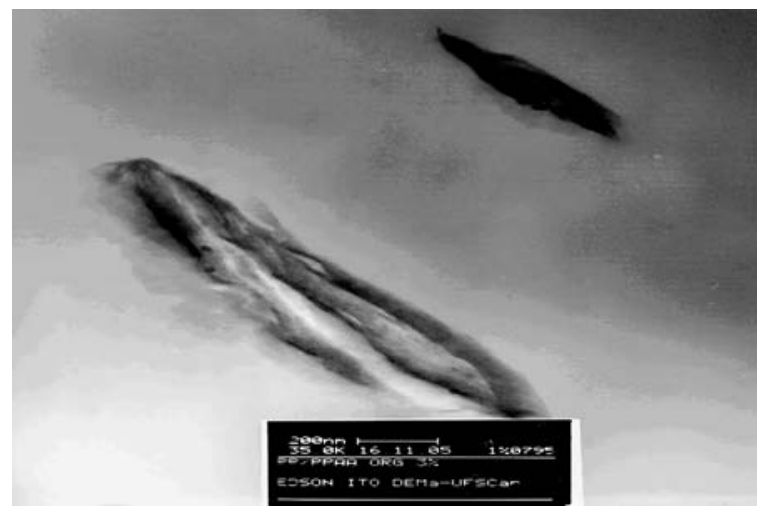

(c)

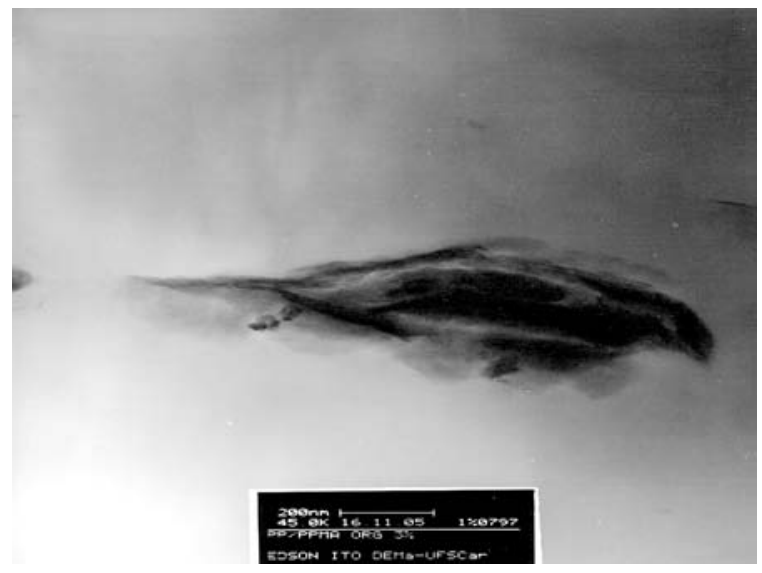

(d)

Figura 5. Micrografia dos sistemas: a) PP/PP-g-AA/ORG 1\%; b) PP/PP-gMA/ORG $1 \%$; c) PP/PP-g-AA/ORG 3\%; e d) PP/PP-g-MA/ORG 3\%. 
PP/PP-g-AA/ORG e PP/PP-g-MA/ORG, com teores de 1 e $3 \%$ em peso de argila organofílica.

Nas micrografias ficou evidente a formação de tactóides e de algumas partículas dispersas. Os tactóides são constituídos de regiões escuras que representam partículas de argila empilhadas e de regiões mais claras que representam a matriz de polipropileno intercalado, ou seja, o polipropileno foi difundido (intercalado) entre as lamelas de argila durante o processamento. No trabalho de Ton et al. ${ }^{[22]}$, os autores atribuem que a difusão é favorecida pela presença de compatibilizantes no sistema.

Portanto, para os sistemas utilizados neste trabalho, o processamento por extrusão não promoveu o cisalhamento necessário para ocorrer uma completa esfoliação/delaminação das lamelas de argila. Neste caso, sugere-se que os nanocompósitos formados apresentam uma estrutura parcialmente intercalada, segundo classificação de Ray \& Okamoto $^{[23]}$.

\section{Propriedades mecânicas dos nanocompósitos}

Nas Tabelas 4, 5, 6 e 7 estão apresentados os resultados dos ensaios mecânicos sob tração para o PP e suas misturas com os compatibilizantes (PP-g-AA e PP-g-MA) e bentonita sódica (SOD) e organofílica (ORG).

A Tabela 4 mostra o resultado do módulo de elasticidade para os sistemas PP/argila, PP/PP-g-AA/argila e PP/PP-g$\mathrm{MA} /$ Argila com 1 e $3 \%$ de argila em peso. Observa-se que o módulo de elasticidade de todos os sistemas apresentou-se próximo ao do PP puro, considerando os erros experimentais. Isto significa que a rigidez do PP não foi alterada com o teor

Tabela 4. Módulo de elasticidade (E) dos materiais.

\begin{tabular}{lcc}
\hline & \multicolumn{2}{c}{ E (MPa) } \\
\cline { 2 - 3 } Material (composição) & $\mathbf{( 1 \% )}$ & $\mathbf{( 3 \% )}$ \\
\hline PP PURO & \multicolumn{2}{c}{$1016 \pm 55$} \\
PP/SOD & $1049 \pm 25$ & $1017 \pm 79$ \\
PP/ORG & $932 \pm 42$ & $951 \pm 52$ \\
PP/PP-g-AA (90/10) & \multicolumn{2}{c}{$1086 \pm 145$} \\
PP/PP-g-MA (90/10) & \multicolumn{2}{c}{$1133 \pm 27$} \\
PP/PP-g-AA/SOD & $973 \pm 22$ & $1003 \pm 31$ \\
PP/PP-g-MA/SOD & $941 \pm 48$ & $1025 \pm 37$ \\
PP/PP-g-AA/ORG & $983 \pm 40$ & $1074 \pm 120$ \\
PP/PP-g-MA/ORG & $994 \pm 39$ & $1070 \pm 39$ \\
\hline
\end{tabular}

Tabela 5. Resistência a tração (RT) dos materiais.

\begin{tabular}{|c|c|c|}
\hline \multirow[b]{2}{*}{ Material (composição) } & \multicolumn{2}{|c|}{ RT (MPa) } \\
\hline & $(1 \%)$ & $(3 \%)$ \\
\hline PP PURO & \multicolumn{2}{|c|}{$23,9 \pm 0,5$} \\
\hline PP/SOD & $29 \pm 0,9$ & $24,5 \pm 0,8$ \\
\hline PP/ORG & $22,4 \pm 0,8$ & $23,0 \pm 0,4$ \\
\hline PP/PP-g-AA (90/10) & \multicolumn{2}{|c|}{$24,6 \pm 0,3$} \\
\hline PP/PP-g-MA (90/10) & \multicolumn{2}{|c|}{$28,1 \pm 0,5$} \\
\hline PP/PP-g-AA/SOD & $24,9 \pm 0,2$ & $24,2 \pm 0,4$ \\
\hline PP/PP-g-MA/SOD & $23,8 \pm 0,7$ & $23,2 \pm 0,4$ \\
\hline PP/PP-g-AA/ORG & $23,4 \pm 0,4$ & $24,3 \pm 0,5$ \\
\hline PP/PP-g-MA/ORG & $26,5 \pm 0,7$ & $26,4 \pm 0,3$ \\
\hline
\end{tabular}

Tabela 6. Percentagem de alongamento final ( $\varepsilon$ ) dos materiais.

\begin{tabular}{lcc}
\hline & \multicolumn{2}{c}{$\varepsilon(\mathbf{\%})$} \\
\cline { 2 - 3 } Material (composição) & $\mathbf{( 1 \% )}$ & $\mathbf{( 3 \% )}$ \\
\hline PP PURO & \multicolumn{2}{c}{$63,2 \pm 9,2$} \\
PP/SOD & $103 \pm 33$ & $191,6 \pm 27,5$ \\
PP/ORG & $268,6 \pm 4,7$ & $285,2 \pm 63,3$ \\
PP/PP-g-AA (90/10) & \multicolumn{2}{c}{$265,5 \pm 67,3$} \\
PP/PP-g-MA (90/10) & \multicolumn{2}{c}{$101,4 \pm 25,6$} \\
PP/PP-g-AA/SOD & $207,5 \pm 68,7$ & $244,2 \pm 57,4$ \\
PP/PP-g-MA/SOD & $332,8 \pm 17,1$ & $288 \pm 70,7$ \\
PP/PP-g-AA/ORG & $>>360$ & $219,1 \pm 73,4$ \\
PP/PP-g-MA/ORG & $231 \pm 47,1$ & $183,8 \pm 48,4$ \\
\hline
\end{tabular}

Tabela 7. Tenacidade (J) dos materiais.

\begin{tabular}{lcc}
\hline & \multicolumn{2}{c}{ Tenacidade (J) } \\
\cline { 2 - 3 } Material (composição) & $\mathbf{( 1 \% )}$ & $\mathbf{( 3 \% )}$ \\
\hline PP PURO & \multicolumn{2}{c}{$34,7 \pm 4,4$} \\
PP/SOD & $16,5 \pm 18.4$ & $106,9 \pm 15,8$ \\
PP/ORG & $128,6 \pm 8,4$ & $150,2 \pm 33,9$ \\
PP/PP-g-AA (90/10) & \multicolumn{2}{c}{$142,9 \pm 36,9$} \\
PP/PP-g-MA (90/10) & \multicolumn{2}{c}{$57,6 \pm 13,7$} \\
PP/PP-g-AA/SOD & $113,3 \pm 35,3$ & $132,3 \pm 29,9$ \\
PP/PP-g-MA/SOD & $172,9 \pm 6,6$ & $150,6 \pm 35,2$ \\
PP/PP-g-AA/ORG & \multicolumn{2}{c}{$>118,3 \pm 39,5$} \\
PP/PP-g-MA/ORG & $129,7 \pm 29,1$ & $102,3 \pm 26,1$ \\
\hline
\end{tabular}

de argila e nem com a organofilização, e nem com tipo de compatibilizante. Na literatura encontram-se diferentes comportamentos em relação ao módulo em sistemas PP/argila organofílica/compatibilizantes. Lopez et al. ${ }^{[13]}$, verificaram comportamento similar ao apresentado nesta pesquisa para o mesmo teor de argila e de compatibilizante utilizados. Em outros trabalhos, foi obtido um aumento maior ( 15\%) em relação à matriz pura de PP quando se utilizou processos de mistura mais eficientes (por ex: extrusoras modulares), concentração maior de compatibilizante e teor de argila organofílica mais elevado entre 5 e $15 \%$ em peso ${ }^{[22,25,26]}$. Nos casos onde ocorreu aumento significativo no módulo, os autores atribuíram a uma maior interação do polímero com a superfície da argila devido a maior intercalação do polímero entre as camadas de argila.

A Tabela 5 mostra o resultado da resistência a tração máxima no ponto de escoamento para os sistemas PP/Argila, PP/PP-g-AA/Argila e PP/PP-g-MA/Argila com 1 e $3 \%$ em peso de argila. Observa-se que a resistência à tração máxima dos sistemas apresentou valor igual ou ligeiramente superior ao do PP puro. Este comportamento é importante uma vez que não foi deteriorada a propriedade de resistência à tração da matriz polimérica. Verificou-se em outros trabalhos desenvolvidos em nanocompósitos PP/argila organofílica que estes valores foram semelhantes e às vezes até inferiores aos obtidos neste trabalho ${ }^{[13,20,21]}$. Valores superiores também foram observados e, da mesma forma que o módulo, somente ocorreu aumento quando se utilizou processos de mistura 
mais eficientes e concentrações maiores de compatibilizante e de $\operatorname{argila}{ }^{[22,25,26]}$.

A Tabela 6 mostra os resultados do alongamento até a ruptura (em percentagem) para os sistemas PP/Argila, PP/PPg-AA/Argila e PP/PP-g-MA/Argila com 1 e $3 \%$ em peso de argila. Observa-se que o alongamento até a ruptura de todos os sistemas com argila, inclusive os sistemas PP/PP-g-AA e PP/PP-g-MA, apresentou valores bem superiores ao do PP puro. O mais surpreendente foi que a adição da argila organofílica aos sistemas PP/PP-g-AA e PP/PP-g-MA contribuíram para elevar ainda mais o alongamento, sobressaindo com o melhor resultado o sistema PP/PP-g-AA/ORG onde todos os corpos de prova ensaiados não romperam dentro do limite da máquina. A explicação para este comportamento não é simples tendo em vista a complexidade dos sistemas. Na literatura sobre nanocompósitos poliméricos é difícil encontrar sistemas que apresentam essa tendência de aumento elevado de ductilidade com a presença de argila. Para o sistema epoxy/ $\operatorname{argila}^{[27]}$ os autores atribuíram esse comportamento ao efeito plastificante dos íons do sal quaternário de amônio presentes entre as camadas da argila, e aos efeitos conformacionais do polímero na interface matriz/argila, porém a explicação para este fenômeno ainda não é conclusiva. Para o comportamento apresentado nos sistemas PP/argila utilizados neste trabalho, acredita-se que os tactóides formados pela intercalação de moléculas do polímero entre as camadas da argila, conforme observado no MET, são delaminados durante o processo de deformação sob tração após o limite de escoamento. Esse mecanismo, fazendo-se uma analogia ao mecanismo de escorregamento de planos cristalinos, provavelmente deverá colaborar para o aumento da ductilidade do material.

A Tabela 7 mostra os resultados de tenacidade (em Joules) para os sistemas PP/Argila, PP/PP-g-AA/Argila e PP/PPg-MA/Argila com 1 e $3 \%$ em peso de argila. Observa-se que a tenacidade de todos os sistemas com argila, inclusive os sistemas PP/PP-g-AA e PP/PP-g-MA, apresentou valores bem superiores ao do PP puro, exceto para o sistema PP/Argila sódica $1 \%$. Observa-se que o sistema PP/PP-g-AA apresenta uma contribuição melhor para elevar a tenacidade que o sistema PP/PP-g-MA. A tenacidade do sistema PP/PP-g-AA/ Argila organofílica não pode ser calculada pois nenhum dos corpos de prova ensaiados foi rompido.

\section{Conclusões}

Os resultados de análise química e granulometria mostraram que o processo de purificação mostrou-se eficiente para redução dos teores dos minerais acessórios e em retirar as frações grosseiras mantendo as finas da argila. Os resultados de FTIR e de DRX mostraram que o sal foi intercalado nos espaços interlamelares da argila sódica tornando-o organofílica. O alargamento dos picos de DRX dos sistemas com argila organofílica para valores de $2 \theta$ menores e a morfologia de tactóides revelada na análise microscópica, indicou a formação de nanocompósitos com estrutura predominante intercalada. Os resultados de propriedades mecânicas mostraram que houve uma melhoria nas propriedades mecânicas alongamento final e tenacidade.

\section{Agradecimentos}

Os autores agradecem ao CNPQ pelo apoio financeiro, à Braskem S/A pelo fornecimento do PP e a Crompton pelo fornecimento dos compatibilizantes: PP-AA e PP-MA e à Clariant pela doação do sal quaternário de amônio Dodigen e a Edson Ito (UFSCar), pelas análises microscópicas no MET.

\section{Referências Bibliográficas}

1. Boesel, L. F. - "Nanocompósitos de Poli(teraftalato de etileno) e Argila" Dissertação de Mestrado, Universidade Federal de São Carlos, Brasil (2001).

2. Wang, K. H.; Choi, M. H.; Koo, C. M.; Choi, Y. S. \& Chung, I. J. - Polymer.; 42, p.9819 (2001).

3. Ray, S. S. \& Okamoto, M. - Progress in Polymer Science., 28, p.1539 (2003).

4. Novak, B. - Adv. Mater., 5, p. 422 (1993).

5. Vaia, R. A. \& Giannelis, E. P.; - Macromolecules., 30, p.8000 (1997).

6. Kaempfer, D.; Thomann, R. \& Mulhaupt, R. - Polymer., 43, p.2909 (2002).

7. Vaia, R. A.; Ishii, H. \& Giannelis, E.P. - Chem. Mater., 5. p.1694 (1993).

8. Vaia, R. A.; Jandt, K. D,; Kramer, E. j. \& Giannelis, E. P.; - Macromolecules., 28, p.8080 (1995).

9. Koh, S. \& Dixon, J. B. - Applied Clay Science., 18. p.111 (2001).

10. Valenzuela-Díaz, F. R. - "Obtenção de Argilas Organofílicas Partindo-se de Argila Esmectíticas e do sal Quaternário de Amônio ARQUAD 2HT-75", in: Anais do $43^{\circ}$ Congresso Brasileiro de Cerâmica, p. 43201, Florianópolis - SC (1999).

11. Valenzuela-Díaz, F. R. - Key Eng. Mat., 189-191, p.203 (2001).

12. Andrade, D. L. A. C. S. - "Desenvolvimento de nanocompósitos polipropileno/bentonita através da técnica de intercalação por fusão", Dissertação de Mestrado, Universidade Federal de Campina Grande, Brasil (2003).

13. García-López, D.; Picazo, O. Merino, J. C. \& Pastor, J. M. - European Polymer Journal., 39, p.945 (2003).

14. Xu, W.; Liang, G.; Zhai, H.; Tang, S.; Hang, G. \& Pan, E. - European Polymer Journal., 39, p.1467 (2003).

15. Tidjani, A.; Wald, O.; Pohl, M. Hentschel. M. P. \& Schartel, B. - Polymer Degradation and Stability., 82. p.133 (2003). 
16. Singh, P.K.; Pankey, A.; Charma, V.P. - Evolution of oil well drilling fluid using yield point and plastic viscosity correlation, Research and Industry, 3 São Paulo (1992).

17. Rodrigues, A. W. B. - "Influência do tratamento da argila bentonita e de compatibilizantes polares no desenvolvimento de nanocompósitos de polipropileno", Dissertação de Mestrado, Universidade Federal de Campina Grande, Brasil (2006)

18. Díaz, V. F. R.; - "Preparação a nível de laboratório de algumas argilas esmectitas organofilicas", Tese de Doutorado, Universidade de São Paulo, Brasil, (1994).

19. Lopez, D. G.; Picazo, O.; Merino, J. C; Pastor, J. M. Polypropylene-clay nanocomposites: effect of compatibilizing agents on clay dispersion. European Polymer Journal, 39, p. 945-950, (2003).

20. Wang, Y.; Chen, F. B.; Li, C. Y. \& Wu, K. C. - Melt processing of polypropylene/clay modified with maleated polypropylene compatibilizes. Composites: Part B, 35, p.111-124 (2004).

21. Lee, K. Y. \& Goettler, L. A.; - Structure-property relationships in polymer blend nanocomposite. Polymer Engineering and Science, 44, 6 (2004).
22. Ton, M.; Perrin, S. F. \& Cole, C, K. - Polyolefin Nanocomposite Formulation and development, Polymer Engineering and science, 44, 7 (2004).

23. Ray, S. S. \& Okamoto, M. - Polymer/Layered silicate nanocomposite: a review from preparation to processing. Progress in Polymer Science, 28, p.1539-1641 (2003)

24. Sousa, S. P. - Ciência e Tecnologia de Argilas. Ed Edgard Blucher Ltda, São Paulo - SP, v1, 1989.

25. Svoboda, P.; Zeng, C.; Wang, H.; Lee, L. J.; Tomasko, D. L. - Morphology and Mechanical Properties of Polypropylene/Organoclay Nanocomposites. Journal of Applied Polymer Science, 85, p.1562-1570 (2002).

26. Liu, X. \& Wu, Q. - PP/Clay nanocomposites prepared by grafting-melt intercalation. Polymer, 42, p.1001310019 (2001).

27. Pinnavaia, T. J. - Beall, G. W. - Polymer-clay nanocomposites, John Wiley Sons, Ltd, New York, (2000).

Enviado: 27/01/07

Reenviado: 03/05/07

Aceito: 18/05/07 